\title{
Differenzierung und Institutionalisierung des Medien- und Kommunikationssystems
}

Donges, Patrick ; Jarren, Otfried

\begin{abstract}
Der Beitrag diskutiert die vielfältigen Formen der Differenzierung und Spezialisierung der publizistischen Medien sowie der Institutionalisierung von Intermediären wie Social-Media-Plattformen für das Konzept Mediensystem. Dabei wird zunächst auf die Probleme des Konzepts Mediensystem eingegangen (Abschnitt 1). Auf der Basis institutionentheoretischer Überlegungen wird sowohl der Ausdifferenzierungsprozess des publizistischen Mediensystems (Mehr-Ebenen-Medien-System) wie dessen Transformation zu einem Kommunikationsund Mediensystem dargestellt und diskutiert. Der Beitrag setzt am Medienbegriff von Ulrich Saxer (1999, S. 6) an, der diese als „komplexe institutionalisierte Systeme um organisierte Kommunikationskanäle von spezifischem Leistungsvermögen“ definiert. Die Ausdifferenzierung der publizistischen Medien, so das in Abschnitt 2 entfaltete Argument, ist ein fortlaufender Prozess, der nicht mit der Entstehung eines abgrenzbaren - zumal nationalstaatlichen - Mediensystems endet. Permanent entstehen neue „organisierte Kommunikationskanäle“, wie etwa Online-Intermediäre oder Plattformen, die immer spezifischere kommunikative Leistungen erbringen oder ermöglichen und neu in das komplexe System institutionalisiert werden müssen. Es entsteht, so die Schlussfolgerung in Abschnitt 3, ein differenziertes und spezialisiertes, sowohl de- als auch neu-institutionalisiertes MehrEbenen-System. Auch wenn sich der Begriff des Mediensystems so stark eingebürgert hat, dass wir ihn vermutlich nie loswerden, wäre es, so unser Fazit in Abschnitt 4, analytisch plausibler, von einem globalen und netzbasiertem Kommunikations- und Mediensystem zu sprechen.
\end{abstract}

DOI: https://doi.org/10.24989/medienjournal.v43i3.1831

Posted at the Zurich Open Repository and Archive, University of Zurich

ZORA URL: https://doi.org/10.5167/uzh-184732

Journal Article

Published Version

Originally published at:

Donges, Patrick; Jarren, Otfried (2020). Differenzierung und Institutionalisierung des Medien- und Kommunikationssystems. Medien Journal, 43(3):27-45.

DOI: https://doi.org/10.24989/medienjournal.v43i3.1831 


\section{Differenzierung und Institutionalisierung des Medien- und Kommunikationssystems}

\section{Abstract}

Der Beitrag diskutiert die vielfältigen Formen der Differenzierung und Spezialisierung der publizistischen Medien sowie der Institutionalisierung von Intermediären wie Social-Media-Plattformen für das Konzept Mediensystem. Dabei wird zunächst auf die Probleme des Konzepts Mediensystem eingegangen (Abschnitt 1). Auf der Basis institutionentheoretischer Überlegungen wird sowohl der Ausdifferenzierungsprozess des publizistischen Mediensystems (Mehr-Ebenen-Medien-System) wie dessen Transformation zu einem Kommunikations- und Mediensystem dargestellt und diskutiert. Der Beitrag setzt am Medienbegriff von Ulrich Saxer (1999, S. 6) an, der diese als „komplexe institutionalisierte Systeme um organisierte Kommunikationskanäle von spezifischem Leistungsvermögen “ definiert. Die Ausdifferenzierung der publizistischen Medien, so das in Abschnitt 2 entfaltete Argument, ist ein fortlaufender Prozess, der nicht mit der Entstehung eines abgrenzbaren - zumal nationalstaatlichen - $\mathrm{Me}$ diensystems endet. Permanent entstehen neue „organisierte Kommunikationskanäle“, wie etwa Online-Intermediäre oder Plattformen, die immer spezifischere kommunikative Leistungen erbringen oder ermöglichen und neu in das komplexe System institutionalisiert werden müssen. Es entsteht, so die Schlussfolgerung in Abschnitt 3, ein differenziertes und spezialisiertes, sowohl de- als auch neu-institutionalisiertes MehrEbenen-System. Auch wenn sich der Begriff des Mediensystems so stark eingebürgert hat, dass wir ihn vermutlich nie loswerden, wäre es, so unser Fazit in Abschnitt 4, analytisch plausibler, von einem globalen und netzbasiertem Kommunikations- und Mediensystem zu sprechen.

Keywords: Mediensystem, Institution, Institutionalisierung, Differenzierung

\section{1 "If you don't know what you are talking about, call it a system"}

Das Konzept des Mediensystems war schon immer mit dem Problem konfrontiert, dass Systeme analytische Konstrukte sind. Es gibt Systeme nicht, sondern wir gehen davon aus, dass es sinnvoll ist, Phänomene als zu einem System zugehörig zu betrachten, sobald diese „Merkmale aufweisen, die es rechtfertigen, den Systembegriff anzuwenden; so wie umgekehrt dieser Begriff dazu dient, Sachverhalte herauszuabstrahieren, 
die unter diesem Gesichtspunkt miteinander und mit andersartigen Sachverhalten auf gleich/ungleich hin vergleichbar sind“ (Luhmann, 1984, S. 16). Das Konzept des Mediensystems ist daher das Ergebnis von empirischen Vergleichen und darauf aufbauenden theoretischen Begründungen, warum eine bestimmte Systemkonzeption sinnvoll ist und andere nicht. Insbesondere in Deutschland ist der Systembegriff eng an die Theorie selbstreferentieller und autopoietischer Systeme von Luhmann gekoppelt, obwohl es auch zahlreiche andere Verwendungszusammenhänge des Systembegriffs und starke Zweifel an der ausschließlichen Orientierung des Mediensystems an einer Selbstreferenz gibt (vgl. Beck, 2015). Die Kommunikationswissenschaft hat sich von diesem engen Verständnis der Systemtheorie freilich gelöst und einen pragmatischeren Weg eingeschlagen: Für den Vergleich von Mediensystemen untereinander „ist es zunächst ausreichend, das Mediensystem als etwas Zusammengesetztes mit komplexen Strukturen und Entwicklungslogiken zu begreifen“, ohne allen „Definitionen und Unterscheidungen [der Systemtheorie] zu entsprechen" (Thomaß, 2007, S. 15). Blum (2014, S. 18) beispielsweise versteht unter Mediensystem „die Gesamtheit öffentlicher und aktueller Medien mit ihren Strukturen, ihrer rechtlichen und ökonomischen Verfasstheit, ihren Freiheiten und Schranken, ihrer Kultur und ihren Traditionen in einem definierten Gebiet". Meist sind diese Gebiete Staaten oder entsprechen anderen politischen Grenzziehungen, was bereits auf die Nähe der medialen und politischen Systemkonzeptionen verweist. Blums Verweis auf „öffentliche und aktuelle Medien“ zeigt zudem, dass das Konzept Mediensystem implizit an den (politischen) Journalismus gekoppelt und unter Medien damit aktuell journalistisch-redaktionell erstellte Angebote verstanden werden. Zudem wird in Forschungen unter dem Konzept Mediensystem stark auf Leitmedien wie Qualitätszeitungen oder den öffentlichen Rundfunk fokussiert, denen eine hohe Relevanz für die politische Kommunikation, den politischen Diskurs, zugeschrieben wird. Wissenschaftliche Fachzeitschriften wurden beispielsweise nie unter dem Konzept Mediensystem subsummiert, obwohl sie die relevanten Medien der Wissenschaft sind. Auch Fachmedien oder Verbandszeitschriften wurden in den Analysen nicht beachtet, obwohl sie für die Artikulation von Interessen wie deren Organisation und der Repräsentation gegenüber anderen Relevanz für politische Meinungs- und Willensbildungsprozesse besitzen.

Das auf die nationale Politik bezogene und pragmatische Verständnis von Mediensystemen war lange Jahre ausreichend, schließlich ist der Systembegriff auch im Alltag eingeführt. Zudem ist die Reduktion von Mediensystemen auf bestimmte Teilbereiche, so eben auf die Leitmedien, für eine komparative Forschungsperspektive sinnvoll. Allerdings ist diese Reduktion dann problematisch, wenn innenpolitische oder gar gesellschaftspolitische Prozesse auf Basis von Medieninhalten untersucht werden. So weist die politische Kommunikationsforschung ein Bias auf: Im Kern geht es empirisch um die publizistischen, aktuellen und universellen Medien und insbesondere dann um die sogenannten politischen Leitmedien. Nur ganz wenige Autorinnen und Autoren haben den Begriff des Mediensystems schon immer abgelehnt. McQuail 
(2005, S. 178) beispielsweise hält das Konzept des Mediensystems für „unable to cope with the diversity of media and changing technology and times", zumal es Medien wie Schallplatten, Kino, Unterhaltungsprogramme etc. gar nicht umfasse. „In most countries today, the media do not constitute a single system with a distinctive philosophy or rational" (ebd.). Durch die mit dem Medienwandel verbundene permanente Erreichbarkeit und Verbundenheit der Nutzerinnen und Nutzer führt zudem dazu, dass die Metapher der Medienumwelt wieder an Bedeutung gewinnt (vgl. u.a. Van Aelst, et al., 2017; Vorderer, Hefner, Reinecke, \& Klimmt, 2017).

Durch den Bezug auf einen eng an die Politik angelehnten Begriff des Mediensystems hat die Kommunikationswissenschaft in den vergangenen Jahren einige Dinge übersehen. Zum Beispiel Veränderungen innerhalb des Mediensystems. Die publizistischen (Massen-) Medien bzw. General Interest-Medien wie auch der Journalismus haben sich in vielfältiger Weise spezialisiert und ausdifferenziert (vgl. u.a. Blöbaum 1994, Jarren, 2015). Die Kommunikationswissenschaft hat ferner klassische Merkmale des journalistischen Mediums Presse wie Aktualität, Periodizität, Universalität, Publizität in unzulässiger Weise auf alle Medien übertragen, obwohl bereits ihre eigenen Medien (wissenschaftliche Fachzeitschriften) diesen Merkmalen offensichtlich nicht entsprechen. Wir sprechen heute von General-Interest- wie auch Special-Interest-Medien und können bei einigen Angeboten sogar von „Very-Special-Interest“ ausgehen, wenngleich eine eindeutige Abgrenzung solcher Kategorien nicht möglich ist (vgl. Abschnitt 3.2). Grenz und Donges (2018) weisen darauf hin, dass weite Teile der in einzelnen Politikfeldern relevanten Kommunikation in Fachmedien stattfinden, die als „alle Formen von Verschriftlichungen (Print- oder Online-Medien) definiert werden [können], die zur Mitteilung sowie Rezeption von themen- oder fachspezifischer Informationen oder Botschaften genutzt werden“. Trotz ihrer hohen Bedeutung zur Vermittlung in und zwischen einzelnen Politikfeldern werden solche Fachmedien beispielsweise bei Inhaltsanalysen, selbst in der politischen Kommunikationsforschung, kaum beachtet.

Mit der Gleichsetzung von politisch relevanten journalistisch-redaktionellen Medien mit allen Medien hat sich die Kommunikationswissenschaft ein Problem eingehandelt. Denn heute gewinnen in Mediensystemen immer mehr Angebote an Bedeutung, die zwar als publizistisch zu bezeichnen sind, aber nicht journalistisch-redaktionell erstellt wurden und sich nicht an die allgemeine Öffentlichkeit richten, sondern an kleine Gruppen bis hin zu einzelnen Personen. Die Kommunikationswissenschaft hat nun wie die Medienpolitik das Problem, (Online-)Intermediäre, Plattformen und die universellen und aktuellen Massen- oder publizistischen Medien zu unterscheiden und entsprechenden Regulierungsrahmen zuordnen zu können (vgl. Dolata, 2018; vgl. Gasser \& Schulz, 2015; Mansell, 2015; Perset, 2010; Rundfunkkommission der Länder, 2018). Das Problem daran zeigt sich bereits anhand der relativ einfachen Frage, ob Alphabet (Google), Amazon oder Facebook Medienunternehmen sind oder nicht. Diese Frage wird sowohl von den Unternehmen selbst als auch in der Wissenschaft sehr unterschiedlich beantwortet (vgl. u.a. Napoli \& Caplan, 2017; Schulz \& Dankert, 2016, S. 
16-17). Im Unterschied zu dem Soziologen Luhmann (1996), der das Mediensystem in die drei Grundformen Nachrichten/Berichte, Werbung und Unterhaltung differenzierte, hat die kommunikationswissenschaftliche Forschung Werbe- und Media-Agenturen auch nie ernsthaft als Teil des Mediensystems betrachtet, sonst wäre sie heute bei der Beantwortung der Frage nicht so zögerlich. Das organisationale Feld der publizistischen Medien, also die Medienorganisationen wie die mit ihnen verbunden weiteren Unternehmen wie Verbänden, wird nicht integral in den Blick genommen. Medien werden lediglich als Medieninhalte, allenfalls noch als Gattungen, begriffen und erfasst. Das war und ist unterkomplex.

Trotz all seiner Probleme scheint der Systembegriff im Fach unersetzbar zu sein, wie gerade das viel beachtete Konzept eines "hybriden Mediensystems" (Chadwick, 2013) zeigt. Chadwick vertritt mit dem Begriff die These, dass wir in der Phase eines Überganges leben, in dem sowohl Regeln des „alten" als auch des „neuen“ Mediensystems gelten und beide Regelsysteme miteinander in Interaktion treten. Die Idee, die Welt nicht mit der einfachen Unterscheidung „entweder-oder" zu betrachten, sondern ein „sowohl-als-auch" anzunehmen, ist nicht neu (vgl. u.a. Beck, 1993, S. 9-23), und das Konzept der Konvergenz wird häufig zur Beschreibung des medialen Wandels eingesetzt (vgl. u.a. Kluth \& Schulz, 2014; Vowe \& Henn, 2015). Es stellt sich aber die Frage, ob die Konzepte der Konvergenz und Hybridität mit den Vorstellungen der Unterscheidbarkeit kompatibel sind, wie sie Systemkonzepten eigen sind. Zu einem Systemverständnis Luhmannscher Prägung, das auf eindeutige Zuordnung von Elementen zu einem System zielt, scheint dies nicht zu passen - entweder etwas ist hybrid, oder es ist ein System.

\section{Differenzierung und Institutionalisierung}

Ein Ausweg aus diesem Dilemma könnte darin bestehen, die Veränderungen von Medien aus einer institutionalistischen Perspektive heraus zu betrachten und diese mit differenzierungstheoretischen Argumenten zu ergänzen (vgl. mit Blick auf Social Media Jarren, 2019). Medien sind Institutionen, d.h. auf Dauer angelegte, durchsetzungsfähige Regelsysteme, die normative Erwartungen schaffen, Mechanismen für ihre Durchsetzung beinhalten, Akteure konstituieren und bei bestehenden Organisationen Wahrnehmung, Präferenzbildung und Strukturen beeinflussen (vgl. Donges, 2006, 2013; Jarren, 2016). Sie wirken damit auf die Gesellschaft nicht nur in Form konkreter Berichterstattung ein, sondern bereits durch ihre Existenz, durch die von Akteuren antizipierte Möglichkeit, Gegenstand von Berichterstattung zu werden. Der institutionelle Zugang macht darauf aufmerksam, dass Medien ihre Wirkung auf die Gesellschaft nicht selbstständig entfalten, sondern diese durch die Wahrnehmung und Interpretation von Akteuren vermittelt wird. Institutionalisierte Regeln weisen auf der einen Seite eine hohe Pfadabhängigkeit auf, können auf der anderen Seite durch das Handeln von Akteuren schrittweise geändert werden. 
An Differenzierungsformen lassen sich mit Luhmann (1977) drei unterscheiden: Segmentäre Differenzierung in gleichartige und gleichrangige Teile, stratifikatorische Differenzierung in ungleichartige und ungleichrangige Teile, sowie funktionale Differenzierung in ungleichartige aber gleichrangige Teile (vgl. auch Schimank, 2000, S. 150-151). Medien, und somit auch Mediensysteme, sind fortlaufend von allen drei Differenzierungsformen tangiert:

- Im Zuge segmentärer Differenzierung steigt die Zahl der Angebote, die insbesondere immer speziellere Interessen und Segmente aufgrund von Individualisierung, Wertewandel und kommunikativen gesellschaftlichen Spezialinteressen bedienen.

- Mit der steigenden Anzahl an Angeboten im Medienbereich steigt der Bedarf an stratifikatorischer Differenzierung, d.h. aus einem „more of the same“ auch qualitative Unterscheidungen zu treffen, etwa zwischen Leit- und Folgemedien. Innerhalb des Mediensystems nehmen Leitmedien in einzelnen Arenen eine führende Stellung ein, an denen sich Folgemedien in ihrer Berichterstattung orientieren. Leitmedien gelten damit sowohl aufgrund ihres eigenen Anspruchs wie in der Außenwahrnehmung (Fremdzuschreibung) als Referenzmedien. Der Begriff Qualitäts- oder Prestigemedien bezieht sich auf die Wertschätzung dieser Medien durch Journalistinnen und Journalisten sowie die Anerkennung der Relevanz publizistischer Leistungen vor allem durch die Elite (vgl. Jarren \& Vogel, 2011).

- Die Spezialisierung im Mediensystem bleibt nicht ohne Folgen für die funktionale Differenzierung. Ein „Dirigieren der Selbstbeobachtung des Gesellschaftssystems“ (Luhmann, 1996, S. 173) lässt sich heute kaum noch als Funktion des Mediensystems formulieren, dafür haben sich sowohl die Gesellschaft insgesamt als auch ihr Mediensystem zu sehr ausdifferenziert. Das Mediensystem stellt keine Selbstbeschreibung der Gesellschaft her oder hält sie allgemein verfügbar, sondern erlaubt lediglich, Selbstbeobachtungsangebote aus unterschiedlichen Positionen zu beobachten (vgl. Marcinkowski, 2002, S. 116).

Die Kommunikationswissenschaft hat zwar das Konzept der Differenzierung immer wieder betont, zugleich aber konkrete Formen an Differenzierung weder theoretisch, vor allem aber nicht empirisch hinreichend bearbeitet. Mit Imhof (2013, S. 9) lässt sich „in den Sozialwissenschaften eine Verkürzung der klassischen Differenzierungstheorie auf funktionale Differenzierung beobachten", die er unter anderem der Adaption der funktionalen Systemtheorie anlastete. Dies habe „zu einer Verdrängung der stratifikatorischen und segmentären Differenzierung und damit auch der Reflexion von Ungleichheit, unterschiedlichen Zugangschancen und Herkunftsattribuierungen sowie ihren Folgen für die soziale Ordnung“ (ebd.) geführt. So kann „Dirigieren der Selbstbeobachtung von Gesellschaft" ${ }^{\text {" }}$ war als Funktion journalistisch-redaktioneller Medien für tages- und allenfalls wochenaktuelle politische Angebote in den nationalstaatlichen Leitmedien plausibel gemacht werden, der Blick allein auf solche Angebote war aber immer schon verkürzt (Jarren, 2015, S. 119). Neben den „klassischen“ journalistischredaktionellen Medien gab es jedoch, wie oben bereits argumentiert, immer schon ein 
breites Angebot an Fachjournalismus (Dernbach, 2010) oder Fachmedien (Grenz \& Donges, 2018), das trotz beeindruckender Auflagen- und Nutzungsdaten kaum beachtet wurde.

Die Prozesse segmentärer, stratifikatorischer und funktionaler Differenzierung sind ursächlich dafür, dass die Unterschiede zwischen universellen und aktuellen Massenmedien, „neuen“ Online-Medien und Plattformen heute immer mehr verschwinden. Dies zeigt sich insbesondere an der fehlenden Trennschärfe der Konzepte Massenmedien, Intermediäre und Plattformen. In der kommunikationswissenschaftlichen Literatur werden die Begriffe unterschiedlich definiert und in Beziehung zueinander gesetzt. Vereinfacht lassen sich zwei Verwendungszusammenhänge unterscheiden: Zum einen wird versucht, die Begriffe voneinander abzugrenzen und insbesondere zwischen Intermediären und den aktuellen, universellen Massenmedien Unterschiede herauszuarbeiten. So definieren beispielsweise Gasser und Schulz (2015, S. 3) Intermediäre als „, in between content and users“, die zwar zur öffentlichen Kommunikation relevante Beiträge leisten, aber keine „traditional journalistic-editorial (,mediac) services" sind. Eine Unterscheidung von Medien und Intermediären scheint insbesondere aus rechtswissenschaftlicher Perspektive wichtig, um die Anwendung unterschiedlicher Regelungsrahmen begründen zu können. In anderen Verwendungszusammenhängen werden dagegen Intermediäre als Oberbegriff gewählt, worunter Medien und Plattformen gefasst werden. Bestimmt man Intermediäre anhand von Funktionszuschreibungen wie „enable communication of information from one party to another" (Cotter, 2006, S. 68) oder „bring together or facilitate transactions between third parties“ (Perset, 2010, S. 9), so lässt sich nicht mehr zwischen universellen und aktuellen Massenmedien unterscheiden.

Die durch Differenzierungsprozesse entstandenen unterschiedlichen Medien weisen einen unterschiedlichen Grad an sowie unterschiedliche Formen von Institutionalisierung auf. Auch wenn einige bezogen auf ihre Kernleistungen als publizistische Medien anzusehen sind: Bezüglich Regeln und Normen für die Selektion, Bereitstellung, Vermittlung (so Formate, Rhythmen u.a.m.) unterscheiden sie sich deutlich voneinander. Das Publikum weiß um diese Unterschiede, kennt also die unterschiedlichen publizistischen Regeln wie Normen. Dies entspricht einer wesentlichen Annahme institutionalistischer Theorien: Akteure - Individuen wie Organisationen - orientieren sich primär an der sozialen Angemessenheit ihres Handelns. Da diese Angemessenheit kein natürlicher oder objektiver Wert ist, folgen sie ihren Wahrnehmungen oder Vermutungen, welches Handeln angemessen sein könnte. In unserem Fall gegenüber den Medien und ihren Regeln.

In einer institutionalistischen Perspektive wird der Medienwandel als Wandel von Regeln sowie als Prozesse der Übernahme dieser Regeln durch Akteure verstanden. Dazu haben bereits DiMaggio und Powell (1983) drei klassische Mechanismen unterschieden: Zwang (auch kultureller Zwang), normativer Druck (vor allem durch Professionsangehörige) sowie Imitation. Man übernimmt etwas, weil man muss, weil man 
von anderen Akteuren dazu gedrängt wird, oder weil man es bei anderen als erfolgreich abgeschaut hat. Institutionen können nur dann entstehen und sich durchsetzen, wenn sie von Akteuren als ,institutional entrepreneurs" unterstützt werden (vgl. DiMaggio, 1988, S. 13; Powell, 1991, S. 191).

Zahlreiche Studien haben darauf aufmerksam gemacht, dass die Übernahme institutioneller Regeln durch Akteure kein einfacher Prozess ist. Zwar benutzen wir auch in der Kommunikationswissenschaft häufig den Begriff der Anpassung. Doch der Medienwandel ist nicht so einfach, wie er durch den Anpassungsbegriff erscheint. Häufig müssen Akteure Anforderungen mehrerer institutioneller Umwelten in Einklang bringen oder verschiedene widersprüchliche Anforderungen zugleich erfüllen. Häufig können Akteure institutionelle Regeln nicht einfach übernehmen, sondern müssen sie übersetzen, mühsam transformieren oder, wie Campbell (2004: 69-74) es formuliert hat, „bricolage“ (übersetzbar mit Handwerksarbeit oder auch Pfusch) betreiben (vgl. Donges, 2013). Von Interesse ist dabei auch, wer die Akteure sind, die institutionellen Wandel vorantreiben, weil sie als "institutionelle Unternehmer" von den (neu etablierten) Regeln profitieren. Dabei ist jedoch eine zeitliche Verschiebung zu berücksichtigen: Institutionen sind in der Regel ein Ausdruck früherer Praktiken und Denkweisen, die heutige Handlungen konditionieren (vgl. Barley \& Tolbert, 1997, S. 99). Wie bereits argumentiert ist unsere heutige Sicht auf das Mediensystem stark durch die universellen und aktuellen Medien geprägt. Für die sich ausdifferenzierenden Medien brauchen sowohl die Gesellschaft als auch die Wissenschaft neue (Leit-) Begriffe.

Im Kern des Konzeptes eines institutionalisierten Mediensystems (wie im Kern des sich herausbildenden Medien- und Kommunikationssystems) sollte aus unserer Sicht der Begriff der Vermittlung stehen. Vermittlung umfasst mehr als der bei Luhmann genannte Begriff der Selbst- oder auch der „Weltbeobachtung" (Saxer, 2013, S. 202). Nicht die Beobachtung ist das entscheidende, sondern die Vermittlung des Beobachteten. Vermittlung lässt sich dann weiter nach einzelnen kommunikativen Grundfunktionen wie Selektion, Aggregation, Artikulation, Beobachtung oder Bewertung differenzieren. Die sozio-technische Form, in der diese Funktionen und Leistungen erbracht werden, erscheint dabei zweitrangig. Dass sich die klassische journalistisch-redaktionelle Form der Vermittlung von einer auf Algorithmen basierten unterscheidet leuchtet ein, die Unterscheidung sollte aber nicht zum Ausgangspunkt einer Konzeptionierung von Mediensystemen gemacht werden. Bereits unter den Bedingungen der Dominanz von Massenmediensystemen wurde bei den Analysen, so auch in komparativer Hinsicht, zu stark im Kern auf die universellen, aktuellen und vorrangig politisch ausgerichteten publizistischen Medien fokussiert. So wurden die Leistungen der weiteren (Massen )Medien, so Zeitschriften oder Fachmedien, für den sozio-politischen wie soziokulturellen Austausch in der Gesellschaft, für die Formulierung von Interessen oder die Aushandlung von Zielen, zu wenig beachtet. 
Mediensysteme können unter den gewandelten sozio-technischen Bedingungen ohnehin nicht mehr allein nationalstaatlich konzipiert werden. Social Media-Plattformen mögen keine Medien im klassischen Sinne sein, sie erbringen aber kommunikative Leistungen für bestimmte Teile der Gesellschaft, ermöglichen Kommunikation, Beobachtung wie Bewertung. Sie sind eigenständig institutionalisiert (vgl. Jarren, 2019). Die Entwicklung des Mediensystems wie auch die Transformation dieses Mediensystems zu einem Medien- und Kommunikationssystem ist als Institutionalisierungsprozess aufzufassen und mit entsprechenden institutionentheoretischen Überlegungen zu verbinden.

\section{Differenzierung des Mediensystems - ein Drei-Ebenen-Modell}

Abbildung 1: Drei-Ebenen-Modell des Kommunikations- und Mediensystems

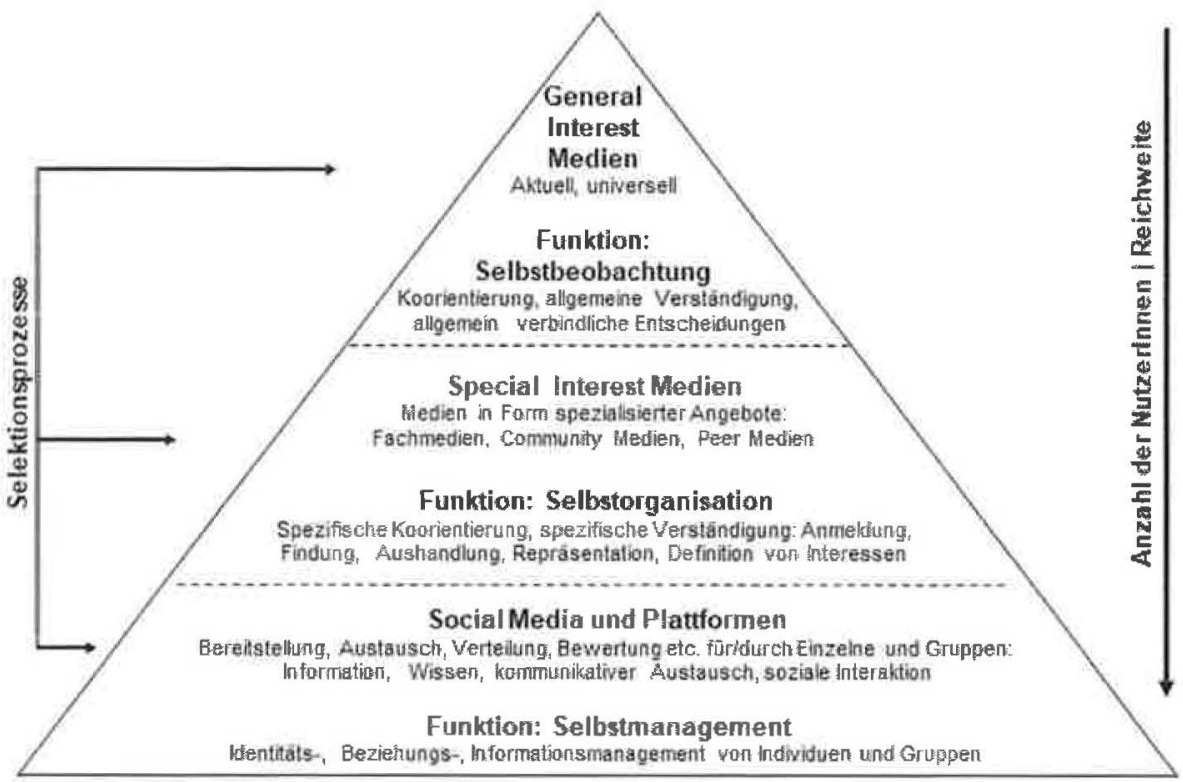

Das publizistische Mediensystem hat sich im historischen Prozess ausdifferenziert. Diese Ausdifferenzierung vollzog und vollzieht sich im Kontext sowohl der stratifikatorischen wie auch der funktionalen gesellschaftlichen Differenzierung. Die aktuellen, universellen Massemedien waren an der Institutionalisierung der Nationalstaaten sowie der mit ihnen etablierten politischen Geltungsräume beteiligt. Diese Medien repräsentieren und reflektieren zugleich auch die funktionale gesellschaftliche Differenzierung, weil sie über die Teilsysteme Politik, Wirtschaft, Sport usw. berichten. Neben den aktuellen, universellen Massenmedien (General Interest Medien) haben sich - im Zuge der neueren segmentären Differenzierung - zugleich immer mehr Special Interest sowie Very Special Interest Media etabliert. Während die aktuellen, uni- 
versellen (Massen-)Medien aufgrund ihrer politischen Bedeutung für die Gesellschaft auch in der Wissenschaft besondere Aufmerksamkeit fanden, gilt dies für die spezialisierten Medien nicht. Damit wurde und wird übersehen, dass auch diese Medien für die gesellschaftliche Entwicklung, für die Findung wie die Organisation von gesellschaftlichen Interessen und damit auch für die politische Meinungs- wie Willensbildung, von Bedeutung waren und sind. Dies zumal aufgrund der zunehmenden gesellschaftlichen Differenzierung, die sich in Individualisierung, Wertepluralismus und Diversität ausdrückt. Mit der Etablierung von Social Media-Plattformen kommt es nun zu einer Neuinstitutionalisierung wie einer weiteren Differenzierung im $\mathrm{Me}-$ diensystem und zur Ausbildung eines globalen Medien- und Kommunikationssystems.

\subsection{General Interest: Die universellen, aktuellen Medien der Gesellschaft}

Vor allem die aktuellen, universellen Medien und der Nationalstaat bildeten bis zur Etablierung des Internets eine Einheit, einen Kommunikationsraum, den man zugleich als das Mediensystem bezeichnete. Diese nationalstaatlichen Kommunikationsräume wurden dabei in der Forschung durch Bezug auf die aktuellen, universellen Medien (Presse sowie Radio und Fernsehen) rekonstruiert und diachron wie synchron miteinander verglichen. Die weiteren publizistischen Medien fanden keine bis wenig Beachtung.

Die universellen, aktuellen Medien sind historisch eng mit den Nationalstaaten verbunden. Sie haben den Nationalstaaten sowohl räumlich (Repräsentation der staatlichen Ebenen, also der politischen Geltungsräume: Bund, Land, Kreis, Gemeinde) als auch sozio-politisch (durch die Repräsentation der sozialen wie politischen Gruppen) eine Gestalt wie ein Gesicht gegeben. Sie haben die politischen Räume wie auch die gesellschaftlichen Machtgruppen repräsentiert. Die Presse, privatwirtschaftlich verfasst, hat sich dabei entlang der jeweils großen gesellschaftlichen Konfliktlinien („links“ "rechts"), institutionalisiert. Dadurch wurde die gesellschaftliche, zumal die politische, Kommunikation vorstrukturiert: Vor allem durch die politisch exponierten Leitmedien innerhalb der Pressestruktur wurde die nationale Gesellschaft sichtbar und dadurch beobachtbar gemacht. Dem öffentlichen Rundfunk wurden mit der Institutionalisierung ebenso entsprechende Vorgaben gemacht: politisch-räumliche wie sozio-politische Repräsentation, zugleich Ausgewogenheit entlang der jeweiligen politischen Machtlinie und gesamtgesellschaftliche Integration als Auftrag. Der Rundfunk wurde deshalb föderal wie binnenplural konzipiert, die privatwirtschaftliche Presse hingegen sollte die gesellschaftliche Außenpluralität ausdrücken. „Publizistische Gewaltenteilung" als ordnungspolitisches Leitbild. Im Nationalstaat institutionalisierte sich damit kulturell wie politisch ein spezifisches Mediensystem, das weitgehend den politischen Geltungsräumen, der politischen Ordnung wie den jeweiligen vorherrschenden gesellschaftlichen Grundpositionen entspricht. Dieses Mediensystem findet in der Mediensystemanalyse vor allem Beachtung. 
Für die Etablierung politischer Institutionen wie die Ausbildung des Nationalstaats und die sozio-kulturelle, die sozio-ökonomische Ausbildung und die Entwicklung von Gesellschaft war die Presse, über lange Zeit getragen von politischen wie gesellschaftlichen Großorganisationen (Kirchen, Gewerkschaften, Verbände), von konstitutiver Bedeutung:

- Mitwirkung an der gesellschaftsweiten Durchsetzung der Schreib- wie Lesefähigkeit im Zusammenspiel mit den Schulen (allgemeine Schulpflicht)

- Förderung der Nationalsprachen, wie aber auch der nationalen Kultur(en)

- Sozialisationsagentur für die Bevölkerung, neben wie nach der Schule

- Ermöglichung der Teilhabe sowie Teilnahme an politischen Prozessen durch die Repräsentation von politischen Geltungsräumen (entsprechend dem Staatsaufbau) und von sozialen Gruppen

- Durch die Bereitstellung von politischen Informationen Mitwirkung an der Schaffung der Voraussetzung für die Institutionalisierung eines allgemeinen und gleichen Wahlrechts

- Durch journalistische Thematisierung und Kommentierung an der Schaffung der Voraussetzung für die Artikulation, Aggregation sowie Durchsetzung von Interessen und somit von neuen gesellschaftlichen Leitbildern im engen Kontext mit gesellschaftlichen Organisationen

Die universellen, aktuellen Massenmedien beziehen sich grundsätzlich und wesentlich auf alle Funktionssysteme der nationalen Gesellschaft. Sie ermöglichen damit die gesellschaftliche Selbstbeobachtung. Entsprechend den Funktionssystemen haben die Medienorganisationen Ressorts innerhalb der Redaktionen institutionalisiert und dementsprechend werden Inhalte journalistisch selektioniert (so Nachrichtenwerte) und strukturiert (so Genres, Formate) angeboten: Politik, Wirtschaft, Kultur, Sport. Durch ihren vorrangigen Bezug auf das System Politik, das allgemein verbindliche Entscheidungen generiert und deshalb für alle Akteure von besonderer funktionaler wie normativer Bedeutung ist, ermöglichen sie einen Mehrsystemblick auf Gesellschaft. Dies auch deshalb, weil die Problemanzeigen aus den gesellschaftlichen Teilsystemen an die Politik als zentrale Lösungsinstanz adressiert werden. Die Journalisten der Massenmedien greifen die Probleme auf (Thematisierung), organisieren, moderieren und bewerten die Debatte und kommentieren Sachverhalte, Institutionen wie Prozesse. Die Probleme der Gesellschaft, die als allgemein lösungsbedürftig angesehen werden, werden wesentlich durch die universellen, aktuellen Massenmedien sichtbar gemacht und können dadurch gesamtgesellschaftlich verfolgt werden (Teilhabe). Dadurch leisten sie eine Systemintegration der Bürgerinnen und Bürger.

Mit dem Konzept der Selbstbeobachtung wird grundsätzlich auf alle Teilsysteme der Gesellschaft abgezielt: Die universellen, aktuellen Massenmedien beobachten vor allem die Kommunikation in den Teilsystemen, die für die Gesamtgesellschaft Relevanz haben. Alle binnenkommunikativen Prozesse können und werden nicht beachtet. Dafür stehen spezialisierte Medien zur Verfügung, die entsprechende Teil- oder 
Zielgruppen lediglich ansprechen. Die aktuellen, universellen Medien hingegen streben eine hohe Reichweite an, haben also einen hohen Inklusions- wie Vergemeinschaftungsanspruch, und sie bedienen sich deshalb einer allgemeinen Selektionslogik und einer allgemeinen (also weniger fachlichen wie fachkommunikativen) Darstellungs- wie Kommunikationsweise. Sie betonen das Allgemeine, Zusammenhängende und weniger das fachlich Spezifische. Sie beziehen sich wesentlich auf laufende Prozesse, bestehende Institutionen und auf die hochaggregierten Interessen. Die Positionen kollektiver wie korporativer Akteure sind für die universellen, aktuellen Medien von großer Bedeutung und sie werden besonders in der Berichterstattung berücksichtigt. Sie reduzieren Komplexität, indem sie den gesellschaftlichen

- Input begrenzen (Relevanz),

- den Throughput im politisch-institutionellen Bereich begleiten (Fokussierung; Transparenz) und

- den Output des politischen Systems vermitteln wie bewerten.

Um ihre hohen Inklusionsziele zu erreichen, sind die universellen, aktuellen Medien in der demokratischen Gesellschaft zudem auf das System Politik fokussiert, denn an dieses Funktionssystem werden alle gesellschaftlichen Probleme adressiert und von diesem System werden allgemeinverbindliche Entscheidungen getroffen. Politische Entscheidungen sind gesellschaftsweit von Bedeutung und Relevanz. Die universellen, aktuellen Massenmedien verfolgen also durch ihren Bezug zur politischen Agenda eine entsprechende Selektionsstrategie gegenüber den Themen aus den Teilsystemen wie aus der Lebenswelt gleichermaßen. Sie leisten durch diese Vermittlung eine Art von gesellschaftlicher Kompaktinklusion: Sie bieten für Viele das allgemein Relevante. Die Massenmedien konstituieren und repräsentieren die funktional differenzierte Gesellschaft. Zur besonderen Leistung der universellen, aktuellen Massenmedien trägt bei, dass sie kostengünstig zu erhalten sind, dass sie viele erreichen wollen und daher Vieles (aber eben nicht Alles) für Alle bieten.

Die Darstellung und Bewertung der gesellschaftlichen Interessenartikulation, der Interessenaggregation sowie die Interessendurchsetzung ist das Kerngeschäft des politischen Journalismus in den Massenmedien. Dadurch soll den Bürgerinnen und Bürgern eine Teilhabe am politischen Prozess ermöglicht werden, ihr Einbezug ist Voraussetzung für bürgerschaftliches Engagement. Zugleich ermöglichen diese Medien den Akteuren die Beobachtung der Interessen, Positionen, Entscheidungen wie Handlungen anderer Akteure. Sie können sich daran orientieren, sich davon abgrenzen, gleich oder anders verhalten. Die General Interest Medien sind deshalb für alle Akteure von Relevanz, weil in ihnen vor allem die kollektiven und korporativen Akteure Beachtung finden. Deren Voten, Positionen, Absichtserklärungen oder Entscheidungen kommt für alle Akteure eine besondere Bedeutung zu, weil sie von deren Entscheidungen betroffen sein können. Die universellen, aktuellen (Massen-)Medien bieten den gesellschaftlichen Akteuren Orientierung, und zwar insbesondere bezogen auf allgemeinverbindliche Entscheidungsprozesse wie die Entscheidungen selbst. 


\subsection{Special Interest: Die spezialisierten Medien der Gesellschaft}

Die moderne Gesellschaft wird immer stärker durch Formen der segmentären Differenzierung geprägt, weil Akteure wie Gruppen im Zuge von Differenzierungsprozessen mehr und mehr ihre Interessen entdecken und zur Geltung bringen: Freisetzung aus Traditionsbindungen, Ent-Traditionalisierung der Lebensführung, Zunahme an räumlicher wie sozialer Mobilität, gewandelte Interessen und neue Formen der Interessenorganisation, neue Arbeits- und folglich auch Freizeitstrukturen (Zeitstrukturen), Zunahme des Selbstgestaltungsanspruches, Geltendmachung unterschiedlicher kultureller Normen wie Werte u. a. m. führen zu Individualisierung und Pluralisierung auf der Mikro-Ebene der Gesellschaft. Peters (1993) bezeichnet diesen Prozess als plurale Differenzierung.

Im Kontext der segmentären Differenzierung der Gesellschaft, verbunden mit Individualisierungs- wie Pluralisierungsentwicklungen in der sich dynamisch entwickelnden Lebenswelt, die zudem von einem steigenden Grad an Mobilität gekennzeichnet ist, übernehmen spezialisierte Medien Funktionen der gesellschaftlichen Selbstorganisation. Die Gestaltung der Lebenswelt wird nämlich anspruchsvoller, weil mehr und mehr auch individuelle Entscheidungen (zumal unter sozial wie auch kognitiv unsicheren Bedingungen) zu treffen sind. Wissen wird für alle Akteure relevanter, gerade auch im Zusammenhang mit persönlichen Entscheidungen. Dieses Wissen können die aktuellen, universellen Medien nur im allgemeinen Masse bieten. Zugleich sind sie weniger auf die jeweiligen Lebenswelten ausgerichtet, da sie sich an den teilsystemischen wie gesamtgesellschaftlichen Prozessen orientieren.

Special Interest-, Very Special Interest-, Fach- sowie Peer-Medien dienen der Interessenfindung, -aushandlung und -repräsentation. Sie ermöglichen sozialen Gruppen binnenkommunikative Selbstverständigungsdiskussionen. Zugleich aber werden durch strukturierende (Markt-) Akteure, so in Form von (Fach-) Zeitschriftenverlagen, entsprechende Konsumgruppen (so für die Werbung) durch spezialisierte Medien konstituiert. Die ökonomische Ausrichtung im Zeitschriftenmarkt, so bei den allgemeinen Publikumszeitschriften, ist wohl Ursache dafür, dass in der Forschung das gesamte Segment wenig Beachtung fand (vgl. Oehmer, Dioh \& Jarren in diesem Heft).

Die spezialisierten Medien, die den Fachjournalismus institutionalisiert haben, dienen - jenseits ihrer Marktfunktionen - der Findung, Aushandlung, Formulierung wie Anmeldung von sozialen, kulturellen, ökonomischen und politischen Interessen (vgl. Weßler, 2002). Interessenfindung sowie -anmeldung erfordern anhaltende (binnen-) kommunikative Verständigungsprozesse, die vor allem von den nicht aktuellen publizistischen Medien geleistet werden. In diesen Prozessen, in denen Wissen in Form vernetzter Informationen entsteht, sind alle Formen von Zeitschriften, aber auch spezialisierte Radio- und Fernsehprogramme - und neu Angebote im Internet - relevant. Diese Angebote sind für die individuelle wie die gesellschaftliche Selbstorganisation bedeutsam. 
Segmentäre Differenzierung darf aber nicht isoliert betrachtet werden: Zwischen den unterschiedlichen gesellschaftlichen Differenzierungsprozessen bestehen Abhängigkeiten:

\begin{abstract}
„Differenzierung steht sowohl für die Emanzipation unterschiedlicher Teile der Gesellschaft voneinander wie für den Verlust gesellschaftlicher Einheit und eindeutiger Zurechenbarkeit; sowohl für effizienzsteigernde Arbeitsteilung und Komplexität als auch für den Verlust an Unmittelbarkeit und Durchschaubarkeit gesellschaftlicher Prozesse; sowohl für die kulturelle Steigerung gesellschaftlicher Teilbereiche, ihrer entfesselten Logiken und ihrer berufsmäßigen Vervollkommnung als auch für ihre kulturelle Verarmung, die Fachmenschentum ohne Geist (Max Weber) hervorbringt, ohne Geist, der für einen einheitlichen Horizont hätte sorgen können; sowohl für die Entstehung pluralistischer Lebenswelten und für die Entlassung letzter Stellungnahmen zur Welt aus unmittelbarem gesellschaftlichen Zugriff als auch für den Verlust sozialer Bindungen und fragloser Zugehörigkeiten" (Nassehi, 1999, S. 12)
\end{abstract}

Vor allem segmentäre Differenzierung kann sowohl als Struktur wie auch als Prozess verstanden werden. Sie vollzieht sich durch Kommunikation und wird, zumal in der „Mediengesellschaft", für den Einzelnen wie die Gesellschaft insgesamt, durch die spezialisierten Medien, die eine Nähe zur Lebenswelt aufweisen, sichtbar und somit konstituiert.

Die spezialisierten Medien konstituieren für Individuen wie Gruppen konkrete und spezielle Kommunikationszusammenhänge, mittels deren sie einerseits ihre Interessen finden, entwickeln und austauschen und mit denen sie andererseits für Organisationen als potentielle Leistungsempfänger erreichbar, ansprechbar werden. So geben Organisationen selbst Publikationen heraus (Corporate Publishing, so von Verbänden, Unternehmen), um die Beziehungen zu ihren Mitgliedern zu pflegen, ihre Interessen öffentlich sichtbar zu machen und aber auch, um neue Mitglieder zu gewinnen. Und spezialisierte Verlage (Zeitschriftenverlage) agieren als strukturierende Akteure, indem sie die Interessen von potentiellen Publika mit den Interessen von Marktorganisationen koppeln. Vor allem die spezialisierten Medien dienen, über den unmittelbaren Zweck der jeweils beteiligten Organisationen hinaus, der Lösung lebensweltlicher Anforderungen von Einzelnen und Gruppen. Sie tragen durch die Bereitstellung spezialisierter Informationen sowie von Wissen, und durch die Teilhabe an spezifischen Diskussionen, so Formen der Binnenkommunikation, zur Selbstorganisation der Gesellschaft bei. Sie ermöglichen und repräsentieren die segmentär differenzierte Gesellschaft. Sie leisten die Sozialintegration der Bürgerinnen und Bürger.

Über alles gesehen hat sich - aufgrund der zunehmenden segmentären Differenzierung - das Mediensystem sukzessive ausdifferenziert und auch von einem anbieterorientierten zu einem nachfrageorientierten System gewandelt. Den Rezipienten stehen in diesem Prozess immer mehr Medien wie Medienangebote zur Verfügung. Sie erhalten im steigenden Maß Auswahlmöglichkeiten, zumal im Bereich der spezialisierten Medien. Der Marktanteil der spezialisierten Medien wächst, während der Marktanteil bei den aktuellen, universellen Medien rückgängig ist. Die Zuwendung zu 
Medien wie deren Nutzung hat sich, nicht zuletzt im Zuge neuer technischer medialer Bereitstellungsformen (Online), geändert (Nutzer; User) (vgl. Babić \& Jandura, 2017). Dies zeigt sich beispielhaft an der niedrigen Zahlungsbereitschaft der Nutzerinnen und Nutzer für Informationsangebote im Netz mit den entsprechenden beobachtbaren ökonomischen Folgen für die Leistungserbringung durch den Journalismus und die Massenmedien.

\subsection{Very Special Interest: Social Media-und Plattformen - Ausdifferen- zierung oder Neuinstitutionalisierung?}

Für das Konzept und das Verständnis von Mediensystem ist relevant, wie die Etablierung von Intermediären als kommunikative Leistungserbringer begriffen und wie diese in Systemkonzeptionen integriert werden sollen. Es handelt sich bei den (neuen) Intermediären nicht um publizistische Medien - und sie selbst sehen sich auch nicht in dieser Leistungsrolle. Plattformen sind auf zwei- oder mehrseitigen Märkten tätig: Sie bringen (Kunden-) Gruppen mit komplementären Bedürfnissen zusammen. Sie wirken damit als Besorgungsdienstleister. Bezeichnet werden damit so unterschiedliche Anbieter wie Suchmaschinen, Soziale Netzwerke, App-Plattformen, UserGenerated-Content-Plattformen, (Micro-)Blogging-Plattformen, News-Aggregatoren oder Verkaufsplattformen (vgl. Schulz \& Dankert, 2016). Akteure können diese Intermediäre für die Beschaffung, Aggregation, Selektion, Präsentation, Weiterleitung oder Bewertung von Informationen und Wissen sowie für Kommunikationszwecke nutzen.

Bezüglich Information und Kommunikation haben sich mit den Social Media neue Intermediäre mit anderen Bereitstellungsformen, Auswahlformaten sowie Inklusionsund Beteiligungsmöglichkeiten in sehr kurzer Zeit etabliert. Sie basieren auf anderen Geschäftsmodellen als die publizistischen Medien (kostenlos, allgemeine Zugänglichkeit, globale Verbreitung, Real-Time-Nutzertargeting in der Werbung) und sie sprechen mit ihren Personalisierungs- und Konfektionsangeboten Individuen gezielt an. Sie ermöglichen Individuen vielfältige Formen an Kollaboration. Sie entsprechen damit den Notwendigkeiten der „Multioptionsgesellschaft“. Aber diese neuen Anbieter sind nicht zu den bestehenden publizistischen Medien hinzugetreten, sie sind nicht Teil der etablierten Medienbranche mit ihren Normen und professionellen journalistischen Standards geworden, sondern setzen sich von ihnen hingegen gezielt ab. Ob es sich bei diesen Marktzutritten lediglich um Marktzutritte neuer Intermediäre oder um eine mediale Neuinstitutionalisierung handelt, soll und kann hier nicht diskutiert werden (vgl. dazu bspw. Jarren, 2019). Zweifellos lösen die neuen Akteure einen fundamentalen institutionellen Wandel im bislang von den publizistischen Medien dominierten und somit geprägten Mediensystem aus. Insbesondere Social Media-Plattformen haben, verbunden mit künstlicher Intelligenz (Algorithmen, Big Data, Deep Learning), 
disruptive Folgen für die publizistischen Medien und deren bislang vorherrschendes gesellschaftliches Vermittlungsmonopol.

Social Media sind nicht nur neue Vermittlungsinstitutionen, sondern sie etablieren zudem auch eine neue - auf ein hohes Maß an Individualität und dynamische Austausch- wie Transaktionsprozesse angelegte - gesellschaftliche Vermittlungsstruktur. Die neuen Social-Media-Plattformen sind keine klassischen, d.h. publizistischen, Medien. Sie ermöglichen Einzelnen, Gruppen, Netzwerken oder Organisationen die Beschaffung und Verbreitung von Informationen jedweder Art. Die neuen Intermediäre in Form der Social Media ermöglichen eine Steigerung der gesellschaftlichen Vermittlungsmöglichkeiten, und zwar sowohl für Individuen, Gruppen, Netzwerke und Organisationen als auch für die Gesamtgesellschaft. Vor allem Individuen - und das ist im Kern die institutionelle Innovation - verfügen über erheblich ausgeweitete Informations-, Kommunikations- und (Selbst-) Organisationsmöglichkeiten mittels dieser Medien. Die neu institutionalisierten Social Media erweitern ebenso die Möglichkeiten zur Selbstbeobachtung wie auch zur Selbstorganisation erheblich. Mit ihnen wird zudem der aktive Mediengebrauch für einzelne Akteure an (einer großen) Öffentlichkeit möglich. Social Media ermöglichen Identitäts-, Beziehungs- wie Informationsmanagement (vgl. Schmidt, 2008). Sie haben eine Selbstmanagementfunktion.

Für die Selbstorganisation der Gesellschaft sind nun internetbasierte Formen der Kommunikation wie Social Media-Plattformen von großer Bedeutung: Vor allem Social Media-Plattformen entsprechen der segmentär differenzierten Gesellschaft: Sie sind Ausdruck, Katalysator und Beschleuniger der segmentären Differenzierung.

\section{Fazit}

Für das Mediensystem als theoretisches wie empirisches Konzept ist wichtig, die Formen der Differenzierung und Institutionalisierung stärker zu berücksichtigen. So kann bei den hier im Vordergrund stehenden publizistischen Medien zwischen den aktuellen, universellen und den spezialisierten Medien unterschieden werden. Zugleich fand in den beiden Sektoren fortlaufend eine Binnendifferenzierung statt. Es ist nach Ursachen für diese Prozesse zu fragen und es sind die jeweiligen institutionellen Formen der Medien, die publizistischen Bereitstellungs-, Vermittlungs- sowie Nutzungsmerkmale (also: die kommunikativen Leistungsmerkmale), vermehrt zu untersuchen. Neben den publizistischen Medien institutionalisieren sich weitere Intermediäre bzw. Vermittler. Das Medien- und Kommunikationssystem ist einerseits von Pfadabhängigkeiten geprägt, beispielsweise bezüglich der Erwartungen, die an die aktuellen und universellen Medien herangetragen werden. Auf der anderen Seite gibt es Brüche, etwa, wenn einzelne Gruppen der Gesellschaft sich in den universellen Medien nicht mehr repräsentiert sehen und eigene Kommunikationsstrukturen aufbauen. So entstehen neue Kommunikationskanäle der Selbst- und Fremdvermittlung wie auch neue Funktionen im sich ausdifferenzierenden globalen Medien- und Kommunikationssystem. 
Aktuelle, universelle Medien wie auch spezialisierte Medien und die neuen Intermediäre sind höchst unterschiedlich organisiert und institutionalisiert. Durch Selbstbeobachtung (Leistung der aktuellen, universellen Medien) und Selbstorganisation (Leistung der spezialisierten Medien wie der Intermediäre) erfolgte bislang eine Form der „Multiinklusion" (Nassehi, 2004, S. 111), so in dem durch die beteiligten Medienwie Kommunikationsorganisationen in unterschiedlicher Weise die Ansprache, Teilhabe und Teilnahme des Publikums, der Ermöglichung von binnen- wie außenkommunikativen Austauschformen, die Bereitstellung sowie der Austausch von Informationen wie Wissen u. a. m. erfolgt. Die Formen der Inklusion bei den publizistischen Medienorganisationen variieren entsprechend ihren Zielsetzungen wie den Inklusionsbedürfnissen der Leistungsabnehmer. Insgesamt gesehen sind die Inklusionsformen eher formal und sehr wenig auf Kollaboration angelegt bei allen publizistischen Medien - und damit unterscheiden sie sich von Social Media. Grundsätzlich aber sind alle publizistischem Medien jedem Gesellschaftsmitglied zugänglich. Die Individuen sind faktisch stets durch multiple Partialinklusionen normativ, kognitiv, sozial und evaluativ in das publizistische Mediensystem - und darüber vermittelt: in die Funktionssysteme der Gesellschaft wie auch in die Sozialsysteme der Lebenswelt - passiv inkludiert. Diese Inklusion erfolgt in der Lebenswelt dabei dominant durch Leistungsbeziehungen zwischen Einzelnen und Medienorganisationen, die durch diese kommunikativ vermittelt sind. Und vor allem (die spezialisierten) Medien sind für die individuelle Selbstorganisation relevant, weil sie eine allgemeine, kommunikative Teilhabe ohne stark formalisierte Regeln (wie Rollen) ermöglichen.

Der Medienwandel wie die Neuinstitutionalisierung von sog. Intermediären, so vor allem Social Media-Plattformen, bildet einen weiteren Schub der Veränderung von Mediensystemen. Aus der „Gesamtheit öffentlicher und aktueller Medien mit ihren Strukturen, ihrer rechtlichen und ökonomischen Verfasstheit, ihren Freiheiten und Schranken, ihrer Kultur und ihren Traditionen in einem definierten Gebiet" (Blum, 2014, S. 18) heraus entsteht ein globales, netzbasiertes Mehr-Ebenen-Medien- und Kommunikationssystem. Der Prozess ist als Transformationsprozess beschreibbar. Die „alten“ publizistischen, journalistisch-redaktionell erstellten Medien bilden eine Ebene des Kommunikationssystems. Sie dienen vorrangig der Bereitstellung von Informationen an ein kollektives Publikum. Mehr noch: Die Öffentlichkeit konstituiert sich in diesem Prozess als Kollektiv. Publizistische Medien tragen einen wesentlichen Anteil daran, dass sich Bürgerinnen und Bürger eines Nationalstaates überhaupt als Kollektiv begreifen.

Diese allgemeine Medienöffentlichkeit wird in dem Mehr-Ebenen-Kommunikationssystem ergänzt durch die Ebene der Special Interest Medien wie der Social Media und Plattformen. Sie sind mit den General Interest Medien in vielfältiger Weise verbunden, so durch Prozesse der Diffusion und der Übernahme von Themen. Die Grenzen der Ebenen sind durchlässig. Durch Special Interest Medien, vor allem durch Social Media und Plattformen, werden vielfältige Formen von Kommunikation mög- 
lich: sowohl global als auch im Nahbereich der Lebenswelt, von Individual-, Gruppen, Organisations- bis hin zu universellen Formen der Massenkommunikation. Den früher auf die Rezipientenrolle festgelegten Individuen wird ein aktiver Mediengebrauch ermöglicht, der Push-Modus der (Massen-)Medien wird um vielfältige Pull-Modi erweitert. Aus dem vormaligen auf Verteilung und Teilhabe angelegten Massenmediensystem wird ein auf Mitwirkung angelegtes globales Medien- und Kommunikationssystem, in dem differenzierte Formen der Vermittlung unter unterschiedlichen institutionellen Anforderungen stattfinden.

\section{Literatur}

Abrahamson, D. (2015). The Future of the Magazine Form: Digital Transformation, Print Continuity. Journal f Magazine and New Media Research, 16(1), 1-6.

Babić, D., \& Jandura, O. (2017). Ein Blick in die Vergangenheil. In O. Jandura, M. Wendelin, M. Adolf, \& J. Wimmer (Hg.), Zwischen Integration und Diversifikation: Medien und gesellschafticher Zusammenhalt im digitalen Zeitalter (S. 105-119). Wiesbaden: Springer Fachmedien.

Barley, S. R., \& Tolbert, P. S. (1997), Institutionalization and Structuration: Studying the Links between Action and Institution. Organization Studies, 18(1), 93-117. doi: 10.1177/017084069701800106

Beck, K. (2015). Systemtheorie / Mediensystem. In J. Krone \& T. Pellegrini (Hrsg.), Handbuch Medienökonomie (S. 1-18). Wiesbaden: Springer Fachmedien.

Beck, U. (1993). Die Erfindung des Politischen. Zu einer Theorie reflexiver Modernisierung. Frankfurt/M.: Suhrkamp.

Blöbaum, Bernd. (1994). Journalismus als soziales System. Geschichte, Ausdifferenzierung und Verselbständigung. Opladen: Westdeutscher Verlag.

Blum, R. (2014). Lautsprecher und Widersprecher: Ein Ansatz zum Vergleich der Mediensysteme. Köln: Herbert von Halem Verlag.

Campbell, J. L. (2004). Institutional Change and Globalization. Princeton, Oxford: Princeton University Press. Chadwick, A. (2013). The Hybrid Media System. Politics and Power. Oxford: Oxford University Press.

Colter, T. F. (2006). Some Observations on the Law and Economics of Inlermediaries. Michigun State Law Review, 67, 67-82.

Czerny, N. (2017). Zeitschriftenverlage zwischen Verdrängung und Verlagerung. Wiesbaden: Springer Fachmedien.

Dernbach, B. (2010). Die Vielfalt des Fachjournalismus Eine systemalische Einführung. Wiesbaden: VS Verlag für Sozialwissenschaften.

DiMaggio, P. J. (1988). Interest and Agency in Institutional Theory. In L. G. Zucker (Hg.), Institutional Patterns and Organizations. Culture and Environment (S. 3-21). Cambridge/Mass.: Ballinger.

DiMaggio, P. J., \& Powell, W. W. (1983). The Iron Cage Revisited: Institutional Isomorphism and Collective Rationality in Organizalional Fields. American Sociological Review, 48(2), 147-160.

Dolata, U. (2018). Privatisierung, Kuratierung, Kommodifizierung. Kommerzielle Plattformen im Internet. SOI Discussion Paper, 2018-04.

Donges, P. (2006). Medien als Institutionen und ihre Aušwirkungen au Organisationen. Perspektiven des soziologischen Neo-Instilutionalismus für die Kommunikationswissenschaft. Medien \&̈ Kommunikationswissenschaft, 54(4), 563-578.

Donges, P. (2013). Klassische Medien als Institutionen. In M. Künzler, F. M. A. Oehmer, M. Puppis, \& C. Wassmer (IIg.), Medien als Institutionen und Organisationen: Institutionalistische Ansätze in der Publizistik- und Kommunikationswissenschaft (S. 87-96). Baden-Baden: Nomos Verlagsgesellschaft mbH \& Co. KG. 
Gasser, U., \& Schulz, W. (2015). Governance of Online Intermediaries: Observations from a Series of National Case Studies. Cambridge, MA: Berkman Klein Center for Internet \& Society (Research Publication No. 2015-5).

Grenz, F., \& Donges, P. (2018). Fachöffentlichkeiten in der politischen Kommunikation. Erkundungen zwischen Medienöffentlichkeit und Policy-Netzwerken. SCM Studies in Communication and Media, 7(3), 392-422. doi: 10.5771/2192-4007-2018-3-392

Imhof, K. (2013). Einleitung. Stratifizierte und segmentierte Öffentlichkeit. In K. Imhof, R. Blum, H. Bonfadelli, \& O. Jarren (Hg.), Stratifizierte und segmentierte Öffentlichkeit (S. 9-14). Wiesbaden: Springer Fachmedien

Jarren, O. (2015). Journalismus - unverzichtbar?! Publizistik, 60(2), 113-122.

Jarren, O. (2016). Nicht Daten, sondern Institutionen fordern die Publizistik-und Kommunikationswissenschaft heraus. Publizistik, 61(4), 373-383.

Jarren, O. (2019). Social Media: Institutionalisierungsprozesse als interdisziplinäre Forschungsaufgabe. Medien Wirtschaft (im Druck).

Jarren, O., \& Vogel, M. (2011). Leitmedien als Qualitätsmedien. Theoretisches Konzept und Indikatoren. In R. Blum, H. Bonfadelli, K. Imhof, \& O. Jarren (Hg.), Krise der Leuchttürme öffentlicher Kommunikation. Vergangenheit und Zukunft der Qualitätsmedien (S. 17-29). Wiesbaden: VS Verlag für Sozialwissenschaften.

Kluth, W., \& Schulz, W. (2014). Konvergenz und regulatorische Folgen. Gutachten im Auftrag der Rundfunkkommission der Länder. Hamburg: Hans-Bredow-Institut (=Arbeitspapiere des Hans-Bredow-Instituts Nr. 30).

Luhmann, N. (1977). Differentiation of Society. The Canadian Journal of Sociology / Cahiers canadiens de sociologie, 2(1), 29-53. doi: 10.2307/3340510

Luhmann, N. (1984). Soziale Systeme. Grundriß einer allgemeinen Theorie. Frankfurt/M.: Suhrkamp.

Luhmann, N. (1996). Die Realität der Massenmedien (2. erw. Aufl.). Opladen: Westdeutscher Verlag.

Lüthy, K. (2013). Die Zeitschrift. Zur Phänomenologie und Geschichte des Mediums. Konstanz-München: UVK.

Mansell, R. (2015). The public's interest in intermediaries. Information Systems Frontiers, 17(6), 8-18. doi: 10.1108/info-05-2015-0035

Marcinkowski, F. (2002). Massenmedien und die Integration der Gesellschaft aus Sicht der autopoietischen Systemtheorie: Steigern die Medien das Reflexionspotential sozialer Systeme?. In K. Imhof, O. Jarren, \& R. Blum (Hg.), Integration und Medien. (S. 110-121). Wiesbaden: Westdeutscher Verlag.

McQuail, D. (2005). Mass Communication Theory An Introduction (5. Aufl.). London, Thousand Oaks, New Delhi: Sage.

Napoli, P. M., \& Caplan, R. (2017). Why media companies insist they're not media companies, why they're wrong, and why it matters. First Monday. doi: http://dx.doi.org/10.5210/fm.v22i5.7051

Nassehi, A. (1999). Differenzierungsfolgen: Beiträge zur Soziologie der Moderne (S. 11-26). Wiesbaden: VS Verlag für Sozialwissenschaften.

Nassehi, A. (2004). Die Theorie funktionaler Differenzierung im Horizont ihrer Kritik. Zeitschrift für Soziologie, 33(2), 98-118.

Perset, K. (2010). The Economic and Social Role of Internet Intermediaries. OECD Digital Economy Papers (171). doi: http://dx.doi.org/10.1787/20716826

Peters, B. (1993). Die Integration moderner Gesellschaften. Frankfurt/M.: Suhrkamp.

Powell, W. W. (1991). Expanding the Scope of Institutional Analysis. In P. J. DiMaggio, \& W. W. Powell (Hg.), The New Institutionalism in Organizational Analysis. (S. 183-203). Chicago, London: University of Chicago Press.

Rundfunkkommission der Länder. (2018). "Medienstaatsvertrag". Diskussionsentwurf zu den Bereichen Rundfunkbegriff, Plattformregulierung und Intermediäre (Juli/August 2018). Abgerufen von https://www. rlp.de/fileadmin/rlp-stk/.../Medienstaatsvertrag_Online_JulAug2018.pdf 
Saxer, U. (1999). Der Forschungsgegenstand der Medienwissenschaft. In J.-F. Leonhard, H.-W. Ludwig, D. Schwarze, \& E. Straßner (Hg.), Medienwissenschaft. Ein Handbuch zur Entwicklung der Medien und Kommunikationsformen. 1. Teilband (S. 1-14). Berlin, New York: Walter de Gruyter.

Saxer, U. (2013). Profil der Publizistik-/Kommunikations-/Medienwissenschaft in institutionalistischer Perspektive. In M. Künzler, F. M. A. Oehmer, M. Puppis, \& C. Wassmer (Hg.), Medien als Institutionen und Organisationen: Institutionalistische Ansätze in der Publizistik- und Kommunikationswissenschaft (S. 191216). Baden-Baden: Nomos.

Schimank, U. (2000). Theorien gesellschaftlicher Differenzierung (2. Aufl.). Opladen: Leske+Budrich.

Schmidt, J. (2008). Was ist neu am Social Web? Soziologische und kommunikationswissenschaftliche Grundlagen. In A. Zerfass, M. Welker, \& J. Schmidt (Hg.), Kommunikation, Partizipation und Wirkungen im Social Web (S. 18-40). Köln: Halem.

Schulz, W., \& Dankert, K. (2016). Die Macht der Informationsintermediäre. Erscheinungsformen, Strukturen, Regulierungsoptionen. Bonn: Friedrich-Ebert-Stiftung.

Thomaß, B. (2007). Einführung. In B. Thomaß (Hg.), Mediensysteme im internationalen Vergleich (S. 7-11). Konstanz: UVK.

Van Aelst, P., Strömbäck, J., Aalberg, T., Esser, F., de Vreese, C., Matthes, J., et al. (2017). Political communication in a high-choice media environment: a challenge for democracy? Annals of the International Communication Association, 41(1), 3-27. doi: 10.1080/23808985.2017.1288551

Vogel, A., \& Holtz-Bacha, C. (Hrsg.). (2002). Zeitschriften und Zeitschriftenforschung. Wiesbaden: Westdeutscher Verlag

Vorderer, P., Hefner, D., Reinecke, L., \& Klimmt, C. (2017). Permanently Online, Permanently Connected: Living and Communicating in a POPC World. London: Routledge.

Vowe, G., \& Henn, P. (2015), „Konvergenz”-Klärung eines kommunikationswissenschaftlichen Schlüsselbegriffs. Kommunikationspolitik für die digitale Gesellschaft (Digital Communication Research 1). doi: http://dx.doi.org/10.17174/dcr.v1.4

Weßler, H. (2002). Multiple Differenzierung und kommunikative Integration - Symbolische Gemeinschaften und Medien. In K. Imhof, O. Jarren, \& R. Blum (Hg.), Integration und Medien (S. 56-76). Wiesbaden: VS Verlag für Sozialwissenschaften. 